Vervloet, M., Dijk, L. van, Santen-Reestman, J., Vlijmen, B. van, Wingerden, P. van, Bouvy, M.L. Bakker, D.H. de. SMS reminders improve adherence to oral medication in type 2 diabetes patients who are real time electronically monitored. International Journal of Medical Informatics: 2012, 81(9), 594-604

\begin{tabular}{l|l} 
Postprint Version & 1.0 \\
\hline Journal website & http://www.sciencedirect.com/science/article/pii/S1386505612000913 \\
\hline Pubmed link & http://www.ncbi.nlm.nih.gov/pubmed/22652012 \\
\hline DOI & $10.1016 / j . j$.jmedinf.2012.05.005
\end{tabular}

This is a NIVEL certified Post Print, more info at http://www.nivel.eu

\title{
SMS reminders improve adherence to oral medication in type 2 diabetes patients who are real time electronically monitored
}

\author{
M. VERVLOET ${ }^{\mathrm{A}}{ }^{*}$, L. VAN DIJK ${ }^{\mathrm{A}}$, J. SANTEN-REESTMAN ${ }^{\mathrm{B}}, \mathrm{B}$. VAN VLIJMEN ${ }^{\mathrm{B}}, \mathrm{P}^{\mathrm{P}}$. \\ VAN WINGERDEN $^{\mathrm{B}}$, M.L. BOUVY ${ }^{\mathrm{C}}$, D.H. DE BAKKER ${ }^{\mathrm{A}, \mathrm{D}}$
}

${ }^{a}$ NIVEL, Netherlands Institute for Health Services Research, P.O. Box 1568, 3500 BN Utrecht, The Netherlands

${ }^{\text {b }}$ Mediq Apotheken, P.O. Box 2450, 3500 GL Utrecht, The Netherlands

${ }^{c}$ Utrecht Institute for Pharmaceutical Sciences, Division of Pharmacoepidemiology and Clinical Pharmacology, Utrecht University, P.O. Box 80082, 3508 TB Utrecht, The Netherlands

\begin{abstract}
A B S T R A C T
Background: Poor adherence to oral antidiabetics has a negative influence on glycaemic control in type 2 diabetes patients. Real Time Medication Monitoring (RTMM) combines real time monitoring of patients' medication use with SMS reminders sent only if patients forget their medication, aiming to improve adherence. This study aimed to investigate the effect of these SMS reminders on adherence to oral antidiabetics in patients using RTMM and investigate patients' experiences with RTMM.

Methods: Data were collected in a RCT involving 104 type 2 diabetes patients with suboptimal adherence to oral antidiabetics. Fifty-six patients were randomised to receive SMS reminders if they forgot their medication, 48 patients received no reminders. Primary outcome measure was adherence to oral antidiabetics registered with RTMM, measured as: (1) days without dosing; (2) missed doses; (3) doses taken within predefined standardized time windows. Patients' experiences were assessed with written questionnaires.

Results: Over the six-month study period, patients receiving SMS reminders took significantly more doses within predefined time windows than patients receiving no reminders: $50 \%$ vs. $39 \%$ within a 1 -h window (p $=0.003)$ up to $81 \%$ vs. $70 \%$ within a 4 -h window $(\mathrm{p}=0.007)$. Reminded patients tended to miss doses less frequently than patients not reminded ( $15 \%$ vs. $19 \%, p=0.065)$. Days without dosing were not significantly different between the groups. The majority of patients reported positive experiences with RTMM and SMS
\end{abstract}


Vervloet, M., Dijk, L. van, Santen-Reestman, J., Vlijmen, B. van, Wingerden, P. van, Bouvy, M.L. Bakker, D.H. de. SMS reminders improve adherence to oral medication in type 2 diabetes patients who are real time electronically monitored. International Journal of Medical Informatics: 2012, 81(9), 594-604

reminders. Conclusion: RTMM with SMS reminders improves adherence of type 2 diabetes patients, especially the precision with which patients follow their prescribed regimen, and is well accepted by patients.

\section{INTRODUCTION}

The prevalence of diabetes is high and increases rapidly. In 2007, 740,000 patients were diagnosed with diabetes in The Netherlands, $90 \%$ of these patients with type 2 diabetes [1]. On the long-term, elevated blood glucose levels in type 2 diabetes patients can cause both macrovascular complications such as cardiovascular disease and stroke, and microvascular complications such as renal disease, blindness, foot ulcerations and nerve damage [1,2]. An important goal in the treatment of type 2 diabetes is to achieve good glycaemic control (lowering the blood glucose to a level close to normal), thereby reducing the incidence of these complications. One of the key strategies in achieving glycaemic control is long-term adherence to oral antidiabetics [3-5]. However, many patients experience difficulties in following the treatment as agreed with the health care provider. Reported adherence to oral antidiabetics ranges from 36\% to 93\% [6]. Although patients' reasons for not following the prescribed treatment are diverse, one of the most commonly reported barriers to adherence in type 2 diabetes patients is forgetfulness $[7,8]$. Reminding patients of their medication intake can help them overcome this barrier $[9,10]$. Studies investigating the effect of reminder packaging (incorporating a date and time for medication to be taken in the package) and audiovisual reminders on medication devices revealed improved adherence rates, but the effects were only modest [1113]. Nowadays, as the number of mobile phone connections exceeds five billion worldwide [14], reminding patients of their medication intake through Short Message Service (SMS) is increasingly being implemented in interventions aimed at improving adherence. SMS allows for medication use, the RTMM system has the unique feature to remind patients only if they forget to take their pills, thus to intervene only when this is necessary. In practice, this means that when a dispenser opening is registered, no SMS reminder is sent, but when the dispenser is not opened in time, the patient receives a SMS as a reminder to take the medication. As such, habituation or a high intrusiveness can be avoided. Two pilot studies involving HIV patients on antiretroviral therapy in Uganda used real time monitoring with Wisepill [20] and the Med-eMonitor [21]. However, the real time monitoring was not combined with an intervention (such as reminding) when patients miss a dose. Both studies did show that these devices were feasible to use and accepted by patients. Our study is, to our knowledge, the first to evaluate this new Real Time Medication Monitoring system with customized SMS-reminding. Furthermore, up to now, no study has investigated the use of SMS reminders to support type 2 diabetes patients in their medication use. Therefore, the main aim of this study is to evaluate the effect of SMS reminders on the adherence to oral medication in type 2 diabetes patients. The use of an electronic monitoring device can already contribute to a higher adherence because of patients' awareness of being monitored [22]. To eliminate this effect, all patients in this study were electronically monitored with RTMM. Patients' experiences with receiving SMS reminders are important aspects when it comes to patients' acceptance of the system and its integration in everyday life. For 
Vervloet, M., Dijk, L. van, Santen-Reestman, J., Vlijmen, B. van, Wingerden, P. van, Bouvy, M.L., Bakker, D.H. de. SMS reminders improve adherence to oral medication in type 2 diabetes patients who are real time electronically monitored. International Journal of Medical Informatics: 2012, 81(9), 594-604

example, patients who perceive the reminders as disturbing are less likely to use RTMM in daily life than patients who perceive the reminders as a support in their medication use. The second aim of this study is thus to assess patients' experiences. t any time, place and setting. As such, SMS-reminding is a relatively simple method with low intrusiveness and relatively low costs [9]. Previous studies evaluating the effect of SMS reminders on adherence showed positive results. Improved adherence rates to e.g. inhaler treatment in asthma patients [15], antiretroviral therapy in HIV patients [16-18], and immunosuppressive medication in liver transplant recipients [19] were demonstrated. In these previous studies, SMS reminders were sent regardless whether or not the medication was taken. This method may cause annoyance and may eventually lead to loss of effectiveness when the automated reminders become a routine. Pop-Eleches et al. found that daily reminders, as opposed to weekly reminders, did not improve adherence to antiretroviral therapy. They considered habituation to a frequent stimulus or intrusiveness of daily messages as possible explanations [18].

In our study, Real Time Medication Monitoring (RTMM) was introduced to avoid sending reminders to patients who already took their medication. RTMM combines electronic monitoring with SMS reminders. Like other electronic monitoring devices such as the Medication Event Monitoring System (MEMS ${ }^{\circledR}$ ), RTMM uses an electronic medication dispenser that records each date and time of dispenser opening at a central server, providing accurate and detailed data on actual medication use. However, RTMM registers these data in real time, making it possible to identify a missed dose as it happens. Because of the real time registration of patients'

\section{METHODS}

\subsection{Design}

Data for this study were collected in a Randomised Controlled Trial (RCT) with two intervention groups and one control group involving type 2 diabetes patients with suboptimal levels of adherence (pharmacy refill rate of their oral antidiabetic medication of less than $80 \%$ ). Patients were recruited in 40 pharmacies belonging to Mediq, a large Dutch pharmacy chain. In both intervention groups, patients received their oral antidiabetics in the RTMM medication dispenser and had their medication use registered in real time during a period of six months. Patients in the control group did not use RTMM. Including this control group enables us to study the effect of the RTMM dispenser itself on patients' adherence by comparing the refill adherence of the control group with that of the group using the dispenser without being reminded. This article describes the impact of SMS reminders on adherence in patients who are electronically monitored, based on the detailed data of patients' medication use registered with RTMM. Since the control group did not use RTMM, no detailed data on their medication use are available. Therefore this article focuses only on the two intervention groups.

Both groups used the electronic dispenser, which sends a brief message to a central server each time it is opened. Each message that is generated by the dispenser is sent wirelessly, through the GSM network, to a central server. This message contains information about the date and time of the medication event (dispenser opening) and 
Vervloet, M., Dijk, L. van, Santen-Reestman, J., Vlijmen, B. van, Wingerden, P. van, Bouvy, M.L., Bakker, D.H. de. SMS reminders improve adherence to oral medication in type 2 diabetes patients who are real time electronically monitored. International Journal of Medical Informatics: 2012, 81(9), 594-604

the identification number of the dispenser. The electronic dispenser works in nearly every country in the world at locations where mobile phones have network coverage. During the intake in the pharmacy, patients were informed about the study by the pharmacy staff, received the electronic dispenser with a brief user instruction, and chose one, two or three (according to their prescribed number of daily doses) time periods within which they would take their medication. These time periods were communicated to the supplier of RTMM, where the software was programmed once accordingly. The medication is placed in the dispenser by the patients themselves. Patients in the first group (from here on called the SMS group) received a SMS reminder if they had not opened their medication dispenser within the agreed time period. The content of the reminder was: "Have you taken your medication yet? Please take your medication as prescribed by your health care provider". In case of forgetting, only one reminder for each dose was sent, to avoid patients becoming annoyed with the alerts. The SMS reminder was sent at the end of the time period. Patients in the second group (in this study functioning as control group and indicated as such through- out this article) received the RTMM medication dispenser but did not receive SMS reminders. At the end of the six-month intervention period, all patients were asked to complete a questionnaire containing questions about their experiences with RTMM. Patients in both groups were asked about the awareness of their medication use and about their accuracy in taking medication since they used RTMM. In addition, patients in the SMS group were asked about their experiences with receiving SMS reminders.

This trial is registered in the Primary Registry of The Netherlands (trial registration number NTR1882). Ethical approval was obtained from the Medical Ethics

Committee of

the Utrecht Academic Medical Centre (METC protocol number 08-165/C). All patients provided written informed consent.

Further details on the study design are described in the trial protocol [23].

\section{[FIGURE 1]}

\subsection{Participants}

Inclusion criteria for participants in the RCT were: (1) using oral antidiabetic medication for at least one year; (2) if insulin was used in combination with oral medication: using insulin for at least six months; (3) having a refill adherence of less than $80 \%$ calculated from pharmacy dispensing data from eleven months preceding the intervention; (4) aged between 18 and 65 years; (5) having collected the last prescription for oral antidiabetics within the two months prior to the intervention; (6) having knowledge of the Dutch language; (7) using a mobile phone. These criteria are elaborated upon in the trial protocol [23]. Patients were selected from 40 Mediq pharmacies, located in both urban and rural areas. The first five selection criteria resulted in a total of 415 patients who were eligible for participation in the study. Within each pharmacy, these patients were randomized (1:1) to either the SMS group $(n=207)$ or the control group $(n=208)$. This randomisation was performed on anonymous data by the research group. The last two criteria, having knowledge of the Dutch language and using a mobile phone, were checked by the pharmacy staff 
Vervloet, M., Dijk, L. van, Santen-Reestman, J., Vlijmen, B. van, Wingerden, P. van, Bouvy, M.L. Bakker, D.H. de. SMS reminders improve adherence to oral medication in type 2 diabetes patients who are real time electronically monitored. International Journal of Medical Informatics: 2012, 81(9), 594-604

when they contacted the patient for participation in the study. Furthermore, the staff checked whether the patient still used oral antidiabetics at that moment. These last criteria led to the exclusion of 43 patients, resulting in 181 patients eligible for participation in the SMS group and 191 in the control group. Ultimately, 104 patients participated in either the SMS group ( $n=56$, net group response: $30.9 \%$ ) or the control group ( $n=48$, net group response: $25.1 \%$ ). A flowchart of participant involvement in this study is shown in Fig. 1. Large differences were seen between pharmacies in the effort they made to invite patients for participation in the study. The number of recruited patients per pharmacy varied strongly, the response ranged from $6 \%$ to $67 \%$ with a median response rate of $25 \%$.

\subsection{Outcome measures}

Adherence to prescribed treatment comprises two aspects: persistence which refers to how long patients follow treatment, and execution which refers to how well patients follow their prescribed regimen [24]. This study focuses on the latter aspect and will be referred to as adherence throughout this article.

The primary outcome measure is adherence to oral antidiabetics, the quality of execution of the prescribed regimen, measured using three different measures:

1. Number of days without dosing, calculated by adding up the days on which no single dose was taken (the dispenser was not opened at all).

2. Proportion of missed doses, which is a measure to assess whether the appropriate number of doses was taken each day. For each day, the number of dispenser openings was subtracted from the number of prescribed daily doses, providing the number of missed doses per day. For example, if a patient on a twice daily dose regimen opened the dispenser only once on a given day, this is counted as one missed dose. If the patient did not open the dispenser at all, two missed doses were counted. The proportion of missed doses was calculated by dividing the total number of missed doses by the total number of prescribed doses during the intervention period.

3. Proportion of doses taken within agreed and predefined standardized time windows, which is a measure to assess the precision with which the patient adheres to the prescribed regimen. The agreed time period between patient and pharmacist that was programmed in the RTMM dispenser varied between patients. To eliminate this variation, standardized time windows were chosen centred around the midpoint of the agreed time window. Four standardized time windows were defined: from one up to $4 \mathrm{~h}$. Doses taken within a standardized time window of $1 \mathrm{~h}$ was defined as doses taken within 30 min before and 30 min after midpoint of the agreed time window. For each of the four standardized time windows, this adherence measure was calculated by dividing the total number of dispenser openings within the time window by the total number of prescribed doses during the intervention period.

These measures were calculated from data registered with the RTMM medication dispenser during the six-month intervention period. 
Vervloet, M., Dijk, L. van, Santen-Reestman, J., Vlijmen, B. van, Wingerden, P. van, Bouvy, M.L. Bakker, D.H. de. SMS reminders improve adherence to oral medication in type 2 diabetes patients who are real time electronically monitored. International Journal of Medical Informatics: 2012, 81(9), 594-604

The secondary outcome measures include patients' response to SMS reminders (impact of the SMS) and patients' experiences with RTMM and with SMS reminders.

To study how quickly patients responded to the SMS in taking their medication, the percentage of doses that patients took within 15 min, within 30 min and within 60 min after the SMS reminder was sent was calculated. Patients' experiences were assessed with a written questionnaire which was handed out to each patient at the end of the intervention. The following aspects were measured:

1. Patients' judgement on their medication use. Patients were asked to rate their level of awareness of their medication use (from lower to higher) and to report the accuracy with which they took their medication (from worse to better) since they used RTMM;

2. Patients' experiences with SMS reminders. This only applied for patients in the SMS group, since patients in the control group did not receive reminders. Patients were asked their level of agreement (from (totally) agree to (totally) disagree) with five statements about the SMS reminders.

The association between patients' evaluation of the reminder system and the number of SMS reminders sent was also studied.

\subsection{Statistical analyses}

In accordance with the intention-to-treat principle, all patients, regardless of whether or not they finished the intervention, were included in the analyses. Differences in the primary outcome measure (multiple measures for adherence) between the two groups were tested with one-tailed T-tests, since we hypothesize that the adherence in the SMS group will be higher than in the control group. Differences between the two groups in background characteristics and in the secondary outcome measures (experiences with RTMM and reminders) were tested with Chi-square test or Fisher's exact test (categorical variables) and T-test (continuous variables). Analyses were performed with Stata version 10.0 for Windows.

\section{. 3 RESULTS}

\subsection{Characteristics of study participants}

A total of 104 type 2 diabetes patients participated in the study, 56 patients in the SMS group and 48 patients in the control group (Fig. 1). No significant differences in baseline characteristics were found between the two groups (Table 1). Patients in both groups were predominantly male and the average age was 55 years. Comedication was comparable between the groups. At baseline, the refill adherence (number of days for which oral antidiabetics were dispensed during the eleven months preceding the intervention divided by the total number of days and multiplied by $100 \%$ ) was $62 \%$ in both groups.

\subsection{Illustration of data registered with RTMM}


Vervloet, M., Dijk, L. van, Santen-Reestman, J., Vlijmen, B. van, Wingerden, P. van, Bouvy, M.L. Bakker, D.H. de. SMS reminders improve adherence to oral medication in type 2 diabetes patients who are real time electronically monitored. International Journal of Medical Informatics: 2012, 81(9), 594-604

Fig. 2 illustrates data on medication use registered with RTMM. Both graphs show data (time and date of medication dispenser opening) of a patient in the SMS group following a medication regimen of two daily doses. Data of patient A (left graph) reveal a regular pattern of medication use. This patient took $87 \%$ of all doses within the agreed time periods and needed 33 SMS reminders during the whole intervention period. Patient B took only $7 \%$ of all doses within the agreed time periods as can been seen from the irregular pattern of medication use shown in the right graph. A total of 195 SMS reminders were sent to this patient.

\section{[FIGURE 2 - TABLE 1]}

\subsection{Early discontinuation of the intervention}

In the SMS and control group, respectively 11 and 14 patients stopped using RTMM before the end of the six-month intervention period. Four patients in the SMS group indicated irregular working hours, poor mobile connection, no longer on oral therapy, or disinterest in using the dispenser as reasons for stopping. Seven stopped without giving a reason. Three patients in the control group gave a reason for discontinuation: inconvenience (two patients) and going abroad. However, 13 dropouts nearly completed the intervention (from five to nearly six months). No significant differences were found between patients who dropped out and patients who completed the intervention, though the baseline refill adherence tended to be lower for dropouts than for patients who completed the six months (58.2\% and $63.2 \%$ respectively, $\mathrm{p}=0.09$ ).

The mean number of days of patients' participation in the intervention was comparable between the two groups: 163 days $(S D=42.7)$ in the SMS group and 165 (SD = 36.9) in the control group. Periods of seven days or longer without opening of the medication dispenser were found in the data of seven patients in the SMS group and ten patients in the control group. These gaps ranged from nine to twelve days in the first group, and seven to forty-five days in the second group.

\subsection{Intervention effects: differences in adherence between the groups}

Table 2 shows adherence levels to oral antidiabetics of the two groups over the intervention period. The average number of days without dosing did not differ significantly between patients who received SMS reminders and patients who did not. Patients receiving SMS reminders took significantly more doses within the predefined standardized time windows, ranging from $50 \%$ of doses taken within a 1$\mathrm{h}$ window up to $81 \%$ within a 4 -h window. In the control group these percentages were $39 \%$ for a 1 -h window up to $70 \%$ within a 4 -h window. Patients who were reminded missed about $5 \%$ less doses than patients who were not reminded ( $\mathrm{p}=$ 0.065). Adherence of patients who completed the whole six-month intervention period (45 and 34 patients in respectively the SMS and control group) was also studied. Similar significant differences in adherence with respect to timing were found between the two groups. Differences according to medication regimen were explored, but no significant differences were found.

\subsection{Number of SMS reminders sent}


Vervloet, M., Dijk, L. van, Santen-Reestman, J., Vlijmen, B. van, Wingerden, P. van, Bouvy, M.L. Bakker, D.H. de. SMS reminders improve adherence to oral medication in type 2 diabetes patients who are real time electronically monitored. International Journal of Medical Informatics: 2012, 81(9), 594-604

Patients received a SMS reminder when they had not opened their dispenser within the agreed time period. Of the $43.3 \%$ of doses not taken within this time period, $7.1 \%$ of doses was taken in the half hour before this period, indicating that patients did not forget their medication but were just too early. To avoid reminding patients who took their pill early, these 30 min were added to the agreed time period. Fig. 3 presents a graphical representation of the number of SMS reminders. Patients received on average 123.1 (SD = 98.0; range: 1-403) SMS reminders and were reminded for $36.2 \%$ of all pre- scribed doses. Taking the prescribed regimen into account, it was found that patients who were prescribed one daily dose $(n=11)$ were reminded most often. They received an average of 62.5 (SD = 44.5; range: $1-127)$ reminders, indicating that they were reminded for $38.6 \%$ of all prescribed doses. Patients following a twice daily dose regimen $(n=25)$ and patients who were prescribed three daily doses $(n=20)$ were reminded for $37.3 \%$ and $33.7 \%$ of prescribed doses respectively, receiving an average of 116.9 (SD = 98.6; range: 8-335) and 164.1 (SD = 102.7; ing few SMS reminders revealed higher adherence levels on all measures described in Table 2, whereas patients receiving many SMS reminders had much lower adherence levels.

\subsection{Impact of the SMS reminder}

The number of doses patients took within a short period of time after they received the SMS reminder can be seen as a direct result of the reminder and gives an indication of the impact of the reminder on patients' medication intake behaviour. Of the forgotten doses for which patients received reminders (on average 123 doses), about 28.3 (26.9\%) doses were taken within 15 min after the SMS was sent. Patients took an additional $7.6 \%$ of doses in the 15 min hereafter, adding up to a total of 37.0 (34.5\%) forgotten doses taken within a half hour after the SMS. Within an hour after the reminder, a total of 48.6 (46.2\%) doses for which patients were reminded were taken, indicating that patients took an additional $11.7 \%$ of doses between 30 and 60 min after the SMS. Patient who were prescribed three daily doses tended to respond the quickest to the SMS reminders, with 30.1\% of reminded doses taken within 15 min after the SMS. On the other hand, patients on a once daily dose regimen tended to have the slowest response.

\section{[FIGURE 3]}

They took 20.4\% doses for which they were reminded within 15 min.

\subsection{Patient experiences with RTMM and SMS}

Respectively 36 (64\%) and 34 (71\%) patients in the SMS and control group completed the post-test questionnaire. In the SMS group, significant differences in percentage of doses taken within the agreed time period $(\mathrm{p}=0.045)$ and within the standardized time windows $(p=0.03)$ were found between patients who responded and patients who did not: patients who completed the questionnaire had higher adherence levels than those who did not. No such differences were found in the control group.

Significantly more patients in the SMS group than the control group reported higher awareness of their medication use (respectively $42.9 \%$ and $18.2 \%, \mathrm{p}=0.041$ ). 
Vervloet, M., Dijk, L. van, Santen-Reestman, J., Vlijmen, B. van, Wingerden, P. van, Bouvy, M.L. Bakker, D.H. de. SMS reminders improve adherence to oral medication in type 2 diabetes patients who are real time electronically monitored. International Journal of Medical Informatics: 2012, 81(9), 594-604

Patients in the SMS group also reported a more accurate intake of their medication (respectively 50.0\% and 26.5\%), although this difference was not significant (Table 3). Nine patients in the SMS group reported that their higher awareness was a direct result of the SMS reminders: seven patients challenged themselves to anticipate the reminder, two patients stated that being reminded made them more aware. The main reason for higher awareness reported by patients in the control group was the fact that their medication use was registered.

The majority of patients in the SMS group had positive experiences with receiving SMS reminders (Table 3). About $75 \%$ of the patients indicated that the reminders supported them in their medication use. Moreover, eight out of ten patients reported that it was good to know that they would be remembered in case they forget their medication. How- ever, $20 \%$ of the patients thought the SMS reminders were disturbing.

\section{[TABLE 3]}

In the SMS group, we also studied whether the number of SMS reminders received by patients was associated with their experiences with the reminder system. Patients who com- pleted the post-test questionnaire received on average less SMS reminders than patients who did not (respectively $110.5(\mathrm{SD}=78.0)$ and $145.7(\mathrm{SD}=125.6)$ reminders), though this difference was not significant due to the large variation ( $\mathrm{p}=$ 0.10). Furthermore, the number of SMS reminders did not differ significantly between patients who had positive experiences with the reminders (e.g. feeling supported by the reminders, appreciating it to be reminded if needed) and patients who did not. Patients who reported a higher awareness of their medication use as a result of using RTMM $(\mathrm{n}=15)$ received significantly more SMS reminders $(\mathrm{M}=$ $142.5, \mathrm{SD}=87.7)$ than patients who did not report so $(\mathrm{M}=84.7, \mathrm{SD}=62.9)(\mathrm{p}=$ 0.03 ). No significant differences were found in the average number of text messages sent between patients who reported a better accuracy in their medication use as a result of using RTMM $(\mathrm{n}=18)$ and patients who did not.

\section{DISCUSSION}

The Real Time Medication Monitoring system tested in this study registers patients' medication use in real time, making it possible to intervene only when this is necessary: only if patients forget to take their medication, they receive a SMS to remind them. This study investigated the effect of RTMM with SMS reminders on the precision with which type 2 diabetes patients adhere to their prescribed oral medication. Our results show that RTMM with SMS reminders has positive effects on patients' adherence to oral antidiabetic medication. Patients who received reminders for forgotten doses took their oral antidiabetics more regularly than patient who were not reminded. In addition, patients who were reminded tended to miss doses less frequently.

The effect of mobile technology on health behaviour has been demonstrated in earlier research. The review of Fjeldsoe et al. showed that SMS-delivered interventions had positive short-term behavioural outcomes [25]. Another review reported that cell phones and text messaging interventions for diabetes selfmanagement care can improve diabetes health outcomes [26]. In these studies, SMS 
Vervloet, M., Dijk, L. van, Santen-Reestman, J., Vlijmen, B. van, Wingerden, P. van, Bouvy, M.L., Bakker, D.H. de. SMS reminders improve adherence to oral medication in type 2 diabetes patients who are real time electronically monitored. International Journal of Medical Informatics: 2012, 81(9), 594-604

was mainly used to facilitate information exchange between patients and health care providers in between clinic visits, e.g. for educational messages, transferring blood glucose levels, and reminders for follow-up visits to the clinic. Lately, SMS is increasingly being used to remind patients of their medication intake. Our findings are in line with the results from previous studies evaluating the effect of daily SMS reminders on adherence. Similar to our results, Strandbygaard et al. found mean adherence rates to asthma medication of $82 \%$ in the group of patients receiving daily SMS reminders and 70\% in the group receiving no reminders [15]. Miloh et al. showed that text messages increased adherence to immunosuppressant medication in liver transplant recipients measured by drug concentrations in the blood [19]. Studies involving HIV-patients revealed: (1) significantly higher adherence in patients who received daily cell phone reminders compared with patients being alerted by a beeper [16], (2) significantly more patients achieving an adherence of more than $95 \%$ in the group of patients receiving SMS reminders than in the group receiving no reminders [17], and (3) a significant increase in adherence resulting from weekly SMS reminders in contrast with daily or no reminders [18]. Due to differences in method of adherence measurement, follow-up period, included patient numbers and patient population, the dimension of reported improvements in adherence in these earlier studies are difficult to compare with our findings. Further- more, these earlier studies all evaluated SMS reminders sent regardless whether the patient had taken the medication or not. Automated daily reminders may cause habituation resulting in loss of effectiveness. This was confirmed by Pop-Eleches et al. who demonstrated that weekly reminders significantly increased adherence in patients with HIV, whereas daily reminders did not [18]. The RTMM system aims at avoiding habituation, by reminding patients only when they need to be reminded. Although Strandbygaard et al. found similar adherence rates with daily reminders, habituation to these automated alerts may not have yet occurred as their follow- up period was only 12 weeks. We followed patients for a longer period of six months. We did not include a group of patients who received automated daily SMS reminders in our intervention. Therefore, we cannot compare the effects of reminders sent daily directly with reminders sent only when needed.

On average, more than one third of doses for which patients received a reminder was taken within 30 min after the SMS, suggesting that these intakes are a direct result of the SMS reminder and indicating the impact of the SMS reminder on patients' behaviour. The number of SMS reminders sent and the impact of the reminder varied between patients. Fig. 2 illustrated these differences. Obviously, not all patients benefit from the reminder system. Our limited patient numbers prevented us from performing subgroup analyses to identify which patients benefit most (or least) from RTMM combined with SMS reminders. Further research with a larger sample is needed to explore these differences between patients. It should also be noted that RTMM with SMS reminders can especially provide a solution for patients who are unintentionally non-adherent, i.e. patients who forget their pills or are inaccurate in their timing [10]. Patients who deliberately miss or alter their doses (intentional nonadherence), for example because of side effects, are not likely to benefit from an intervention based on reminding. Although our inclusion criteria were aimed at including unintentionally non-adherent patients, our sample may include intentionally non-adherent patients for whom the intervention provided no solution. 
Vervloet, M., Dijk, L. van, Santen-Reestman, J., Vlijmen, B. van, Wingerden, P. van, Bouvy, M.L., Bakker, D.H. de. SMS reminders improve adherence to oral medication in type 2 diabetes patients who are real time electronically monitored. International Journal of Medical Informatics: 2012, 81(9), 594-604

Results from patient questionnaires suggest that RTMM with SMS reminders made patients more aware and more accurate in their medication use. The majority of patients had positive experiences with the SMS reminders, they considered the reminders as useful and a support in their medication use. These findings are in line with other studies that reported on the acceptability of electronic reminders [27,28]. It should be noted that patients in the SMS group who completed the post-test questionnaire had higher adherence rates than patients who did not return the questionnaire. Patients with higher adherence may be more likely to hold a positive attitude to the system. Interestingly, patients receiving many reminders were not more likely to have negative experiences with the reminders than patients receiving few reminders.

We collected no clinical data (i.e. blood glucose levels) in our study. RTMM aims to support patients in their medication use, by sending SMS reminders if they forget their medication. Our intervention aimed primarily on improving adherence. Therefore, the aim of our study was to evaluate the effect of the SMS reminders on the adherence of type 2 diabetes patients. Long- term treatment with oral antidiabetics is one of the factors that contribute to achieving glycaemic control (maintaining blood glucose levels to an normal range), which is the main health outcome for type 2 diabetes patients [3-5]. Although adherence appears to be an intermediate outcome measure, several studies provided evidence of an association between adherence to oral antidiabetics and improved glycaemic control [5,29]. Nevertheless, further research is recommended to investigate the impact of RTMM and SMS reminders on glycaemic control.

A common critique of electronic monitoring is that it cannot be confirmed that the medication is actually taken or that no more or no less than the prescribed dose is taken. Only drug assays can confirm ingestion. How- ever, studies comparing drug assays with medication intake behaviour measured through electronic dispensers confirmed the validity of electronic monitoring devices [29-31]. Further- more, electronic monitoring devices register detailed data on actual medication use and as such provide an objective and reliable measure of adherence [32]. Most electronic monitoring devices give no direct access to data on patients' medication use, making it impossible to react directly to patients' behaviour. RTMM is one of the first systems to provide this possibility.

\subsection{Limitations of the study}

A limitation of our study is the small sample size. As a result, our study has insufficient power to perform subgroup anal- ysis. One explanation for the low response rate may be that we only included patients with suboptimal levels of adherence. These patients appear to be more difficult to include in adherence studies, possibly because of a lower interest in (good) medication use. However, one of the recommendations of a metareview was to concentrate on patients with suboptimal levels of adherence [33]. These patients can benefit most from interventions aimed at improving adherence. A second explanation may be a the occurrence of a language barrier. All materials, e.g. the instructions on how to use the RTMM dispenser, the information about the study and the questionnaires, were written in Dutch. Part of the participating pharmacies were located in urban areas where many immigrants live. Non-Western immigrants were in principle an important target population in this study: the prevalence of diabetes is higher for non-Western immigrants than for the 
Vervloet, M., Dijk, L. van, Santen-Reestman, J., Vlijmen, B. van, Wingerden, P. van, Bouvy, M.L., Bakker, D.H. de. SMS reminders improve adherence to oral medication in type 2 diabetes patients who are real time electronically monitored. International Journal of Medical Informatics: 2012, 81(9), 594-604

indigenous population [34] and non-Western immigrants have lower adherence rates [35]. Making trial materials culturally sensitive, e.g. making patient information and questionnaires available in other languages, might have led to higher participation [36]. Nonetheless, a quarter of the patients in our study are non-Western immigrants. A third explanation for the low response rate may be the influence of individual pharmacies on the recruitment of patients. We found large differences in response rate between pharmacies, which may be explained by differences in the amount of energy the pharmacy staff put into following up on patients for inclusion in the trial. Monetary incentives for both the pharmacy (for contacting patients) and the patient (for participation in the trial) might have improved the response rate [36]. Finally, patients had to make an extra visit to the pharmacy to receive the RTMM dispenser and its corresponding user information. Preventing these extra visits by providing the dispenser and additional information when the patient had to visit the pharmacy anyway to refill their medication, might have led to higher participation. Despite the low response rate, the baseline characteristics of patients were comparable between the two groups.

Another limitation of the study may be that we have not included an arm in the RCT in which patients used RTMM and received daily SMS reminders regardless of whether they had already taken their medication. This would have provided information on the difference in effect between automated daily SMS reminders and our customized SMS reminders. However, this would mean recruiting more pharmacies to include more patients, lowering the feasibility of the study.

\subsection{Further implications}

This study focused on the precision with which patients adhere to their prescribed regimen, observed from the detailed data as registered by RTMM. Since an electronic monitoring itself can already contribute to a higher adherence [22], in our future study we will investigate differences in refill adherence to oral antidiabetics between patients who use RTMM with and without SMS reminders and patients who do not use the RTMM system at all.

The real time registration of patients' medication use may also provide opportunities for health care providers to support patients in their medication use. For example, the general practitioner, pharmacist or diabetes nurse can intervene when they observe directly from the detailed data on medication use that a patient is not taking his/her medication. These data give them an opportunity to start the conversation about adherence with the patient at that moment that a patient experiences difficulties in adhering to the prescribed treatment.

\section{CONCLUSION}

Type 2 diabetes patients who use Real Time Medication Moni- toring (RTMM) and receive SMS reminders if they forget to take their medication have higher adherence levels with respect to timing than patients who are only monitored with RTMM. Overall, patients' experiences with the RTMM system are pos- itive. Since other patient groups have difficulties in following prescribed therapy as well, the RTMM system may provide opportunities for increasing adherence to medication for other chronic diseases. 
Vervloet, M., Dijk, L. van, Santen-Reestman, J., Vlijmen, B. van, Wingerden, P. van, Bouvy, M.L. Bakker, D.H. de. SMS reminders improve adherence to oral medication in type 2 diabetes patients who are real time electronically monitored. International Journal of Medical Informatics: 2012, 81(9), 594-604

\section{Authors' contributions}

LvD and JS designed the Randomised Controlled Trial. MV, LvD, JS and BvV were involved in the implementation of the study. MV prepared the data and performed the analyses. MV, LvD, MB and DdB contributed to the interpretation of the data. MV drafted the manuscript. LvD, JS, BvV, PvW, MB and DdB criti- cally revised the manuscript. All authors read and approved the final version of the manuscript.

\section{Conflict of interest}

The author(s) declare that they have no conflict of interest.

\section{Acknowledgements}

This study was supported by an unrestricted grant from Achmea Healthcare Foundation. The RTMM system was pro- vided by Evalan and partly financed by Achmea Healthcare Foundation. Both Achmea Healthcare Foundation and Evalan had no role in the study design, nor in analysis and interpre- tation of the data, nor in the writing of this manuscript.

The authors would like to thank the pharmacies and the patients for their participation in this study

\section{SUMMARY POINTS}

What was already known on the topic:

- Adherence to oral antidiabetics is low.

- Mobile phone technology can be successful in improving health behaviour.

What this study adds to our knowledge:

- Real Time Medication Monitoring provides detailed and accurate data on patients' medication use.

- SMS reminders are useful in improving adherence to oral antidiabetics, especially the precision with which patients adhere to their prescribed regimen.

- Receiving SMS reminders is appreciated by patients.

\section{R E F E R E N C E S}

[1] C.A. Baan, C.G. Schoemaker, M.A.M. Jacobs-van der Bruggen, H.H. Hamberg-van Rheenen, H. Verkleij, S. Heus, et al., Diabetes up to 2025. Prevention, Treatment and Care Integrated (in Dutch: Diabetes tot 2025. Preventie en zorg in samenhang), National Institute for Public Health and the Environment, Bilthoven, 2009.

[2] G.E. Dailey III, Improving oral pharmacologic treatment and management of type 2 diabetes, Manag. Care 13 (2004) 41-47.

[3] J.M. Schectman, M.M. Nadkarni, J.D. Voss, The association between diabetes metabolic control and drug adherence in an indigent population, Diabetes Care 25 (2002) 10151021.

[4] D.B. Lawrence, K.R. Ragucci, L.B. Long, B.S. Parris, L.A. Helfer, Relationship of oral antihyperglycemic (sulfonylurea or metformin) medication adherence and hemoglobin A1c goal attainment for $\mathrm{HMO}$ patients enrolled in a diabetes disease management program, $\mathrm{J}$. Manag. Care Pharm. 12 (2006) 466-471.

[5] Y. Rozenfeld, J.S. Hunt, C. Plauschinat, K.S. Wong, Oral antidiabetic medication adherence and glycemic control in managed care, Am. J. Manag. Care 14 (2008) 71-75. 
Vervloet, M., Dijk, L. van, Santen-Reestman, J., Vlijmen, B. van, Wingerden, P. van, Bouvy, M.L. Bakker, D.H. de. SMS reminders improve adherence to oral medication in type 2 diabetes patients who are real time electronically monitored. International Journal of Medical Informatics: 2012, 81(9), 594-604

[6] J.A. Cramer, A systematic review of adherence with medications for diabetes, Diabetes Care 27 (2004) 1218-1224.

[7] E.A. Walker, M. Molitch, M.K. Kramer, S. Kahn, Y. Ma, S. Edelstein, et al., Adherence to preventive medications: predictors and outcomes in the Diabetes Prevention Program, Diabetes Care 29 (2006) 1997-2002.

[8] P.S. Odegard, S.L. Gray, Barriers to medication adherence in poorly controlled diabetes mellitus, Diabetes Educ. 34 (2008) 692-697.

[9] M. Cocosila, N. Archer, A framework for mobile healthcare answers to chronically ill outpatient non-adherence, Inform. Prim. Care 13 (2005) 145-152.

[10] A.L. Wroe, Intentional and unintentional nonadherence: a study of decision making, J. Behav. Med. 25 (2002) 355-372.

[11] C.J. Heneghan, P. Glasziou, R. Perera, Reminder packaging for improving adherence to self-administered long-term medications, Cochrane Database Syst. Rev. (2006) CD005025.

[12] T. Charles, D. Quinn, M. Weatherall, S. Aldington, R. Beasley, S. Holt, An audiovisual reminder function improves adherence with inhaled corticosteroid therapy in asthma, J. Allergy Clin. Immunol. 119 (2007) 811-816.

[13] A. Christensen, L.L. Christrup, P.E. Fabricius, M. Chrostowska, M. Wronka, K. Narkiewicz, et al., The impact of an electronic monitoring and reminder device on patient compliance with antihypertensive therapy: a randomized controlled trial, J. Hypertens. 28 (2010) 194-200.

[14] BBC News Technology. Over 5 billion mobile phone connections worldwide. July 9 2010. http://www.bbc.co.uk/news/10569081 (lastly accessed 5.05.11), 2010.

[15] U. Strandbygaard, S.F. Thomsen, V. Backer, A daily SMS reminder increases adherence to asthma treatment: a three-month follow-up study, Respir. Med. 104 (2010) 166-171.

[16] H. Hardy, V. Kumar, G. Doros, E. Farmer, M.L. Drainoni, D. Rybin, et al., Randomized controlled trial of a personalized cellular phone reminder system to enhance adherence to antiretroviral therapy, AIDS Patient Care STDS (2011).

[17] R.T. Lester, P. Ritvo, E.J. Mills, A. Kariri, S. Karanja, M.H. Chung, et al., Effects of a mobile phone short message service on antiretroviral treatment adherence in Kenya (WelTel Kenya1): a randomised trial, Lancet 376 (2010) 1838-1845.

[18] C. Pop-Eleches, H. Thirumurthy, J.P. Habyarimana, J.G. Zivin, M.P. Goldstein, D. de Walque, et al., Mobile phone technologies improve adherence to antiretroviral treatment in a resource-limited setting: a randomized controlled trial of text message reminders, AIDS 25 (6) (2011) 825-834.

[19] T. Miloh, R. Annunziato, R. Arnon, J. Warshaw, S. Parkar, F.J. Suchy, et al., Improved adherence and outcomes for pediatric liver transplant recipients by using text messaging, Pediatrics 124 (2009) e844-e850.

[20] J.E. Haberer, J. Kahane, I. Kigozi, N. Emenyonu, P. Hunt, J. Martin, et al., Real-time adherence monitoring for HIV antiretroviral therapy, AIDS Behav. 14 (2010) 1340-1346.

[21] J.E. Haberer, G.K. Robbins, M. Ybarra, A. Monk, K. Ragland, S.D. Weiser, et al., Realtime electronic adherence monitoring is feasible comparable to unannounced pill counts, and acceptable, AIDS Behav. 16 (2) (2012) 375-382.

[22] G.E. Wetzels, P.J. Nelemans, J.S. Schouten, C.D. Dirksen, W.T. van der, H.E. Stoffers, et al., Electronic monitoring of adherence as a tool to improve blood pressure control. A randomized controlled trial, Am. J. Hypertens. 20 (2007) 119-125.

[23] M. Vervloet, L. van Dijk, J. Santen-Reestman, B. van Vlijmen, M.L. Bouvy, D.H. de Bakker, Improving medication adherence in diabetes type 2 patients through Real Time Medication Monitoring: a randomised controlled trial to evaluate the effect of monitoring patients' medication use combined with short message service (SMS) reminders, BMC Health Serv. Res. 11 (2011) 5.

[24] J. Urquhart, B. Vrijens, New findings about patient adherence to prescribed drug dosing regimens: an introduction to pharmionics, Eur. J. Hosp. Pharm. Sci. 11 (2005) 103-106.

[25] B.S. Fjeldsoe, A.L. Marshall, Y.D. Miller, Behavior change interventions delivered by mobile telephone short-message service, Am. J. Prev. Med. 36 (2009) 165-173.

[26] S. Krishna, S.A. Boren, Diabetes self-management care via cell phone: a systematic review, J. Diabetes Sci. Technol. 2 (2008) 509-517. 
Vervloet, M., Dijk, L. van, Santen-Reestman, J., Vlijmen, B. van, Wingerden, P. van, Bouvy, M.L. Bakker, D.H. de. SMS reminders improve adherence to oral medication in type 2 diabetes patients who are real time electronically monitored. International Journal of Medical Informatics: 2012, 81(9), 594-604

[27] L. Sahm, A. MacCurtain, J. Hayden, C. Roche, H.L. Richards, Electronic reminders to improve medication adherence-are they acceptable to the patient? Pharm. World Sci. 31 (2009) 627-629.

[28] A. Christensen, L.L. Christrup, P.E. Fabricius, M. Chrostowska, M. Wronka, K. Narkiewicz, et al., Survey of patient and physician assessment of a compliance reminder device in the treatment of hypertension, Blood Press. 18 (2009) 280-285.

[29] B. Vrijens, E. Goetghebeur, The impact of compliance in pharmacokinetic studies, Stat. Methods Med. Res. 8 (1999) 247-262.

[30] P. Girard, L.B. Sheiner, H. Kastrissios, T.F. Blaschke, Do we need full compliance data for population pharmacokinetic analysis? J. Pharmacokinet. Biopharm. 24 (1996) 265-282.

[31] B. Vrijens, E. Tousset, R. Rode, R. Bertz, S. Mayer, J. Urquhart, Successful projection of the time course of drug concentration in plasma during a 1-year period from electronically compiled dosing-time data used as input to individually parameterized pharmacokinetic models, J. Clin. Pharmacol. 45 (2005) 461-467.

[32] K.C. Farmer, Methods for measuring and monitoring medication regimen adherence in clinical trials and clinical practice, Clin. Ther. 21 (1999) 1074-1090.

[33] S. van Dulmen, E. Sluijs, L. van Dijk, D. de Ridder, R. Heerdink, J. Bensing, Patient adherence to medical treatment: a review of reviews, BMC Health Serv. Res. 7 (2007) 55.

[34] Statistics Netherlands. Four percent of the Dutch population has diabetes [in Dutch: Van de Nederlanders heeft 4 procent suikerziekte]. http://www.cbs.nl/nlNL/menu/themas/gezondheid- welzijn/publicaties/artikelen/archief/2009/2009-2718wm.htm (lastly accessed 18.03.10).

[35] L. van Dijk, E.R. Heerdink, D. Somai, S. van Dulmen, E.M.Sluijs, D.T. de Ridder, et al., Patient risk profiles and practice variation in nonadherence to antidepressants, antihypertensives and oral hypoglycemics, BMC Health Serv. Res. 7 (2007) 51.

[36] J.M. Watson, D.J. Torgerson, Increasing recruitment to randomised trials: a review of randomised controlled trials, BMC Med. Res. Methodol. 6 (2006) 34. 
Vervloet, M., Dijk, L. van, Santen-Reestman, J., Vlijmen, B. van, Wingerden, P. van, Bouvy, M.Ln Bakker, D.H. de. SMS reminders improve adherence to oral medication in type 2 diabetes patients who are real time electronically monitored. International Journal of Medical Informatics: 2012, 81(9), 594-604

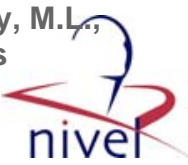

FIGURE AND TABLE.

FIG. 1 - FLOWCHART OF PARTICIPANT INVOLVEMENT IN THIS STUDY.

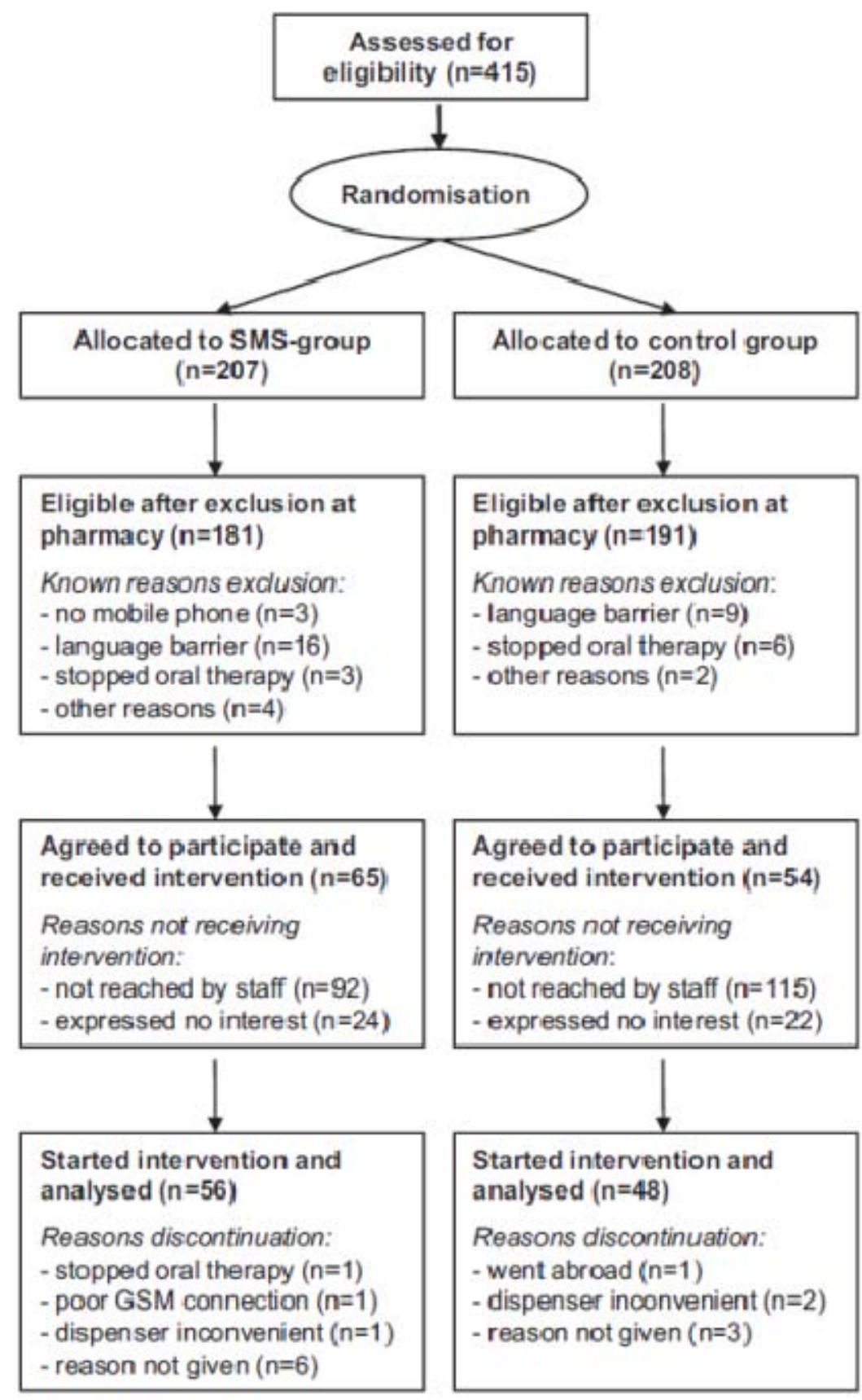

FIG. 2 - TWO EXAMPLES OF MEDICATION INTAKE DATA AS

REGISTERED WITH RTMM. THE HORIZONTAL AXIS DISPLAYS THE DAYS

DURING THE INTERVENTION, THE VERTICAL AXIS DISPLAYS THE $24 \mathrm{H}$

OF EACH DAY. THE DOTS REPRESENT THE MOMENT OF MEDICATION

DISPENSER OPENING, THE HORIZONTAL DOTTED LINES REPRESENT

THE AGREED TIME PERIODS. 
Vervloet, M., Dijk, L. van, Santen-Reestman, J., Vlijmen, B. van, Wingerden, P. van, Bouvy, M.L/M Bakker, D.H. de. SMS reminders improve adherence to oral medication in type 2 diabetes patients who are real time electronically monitored. International Journal of Medical Informatics: 2012, 81(9), 594-604
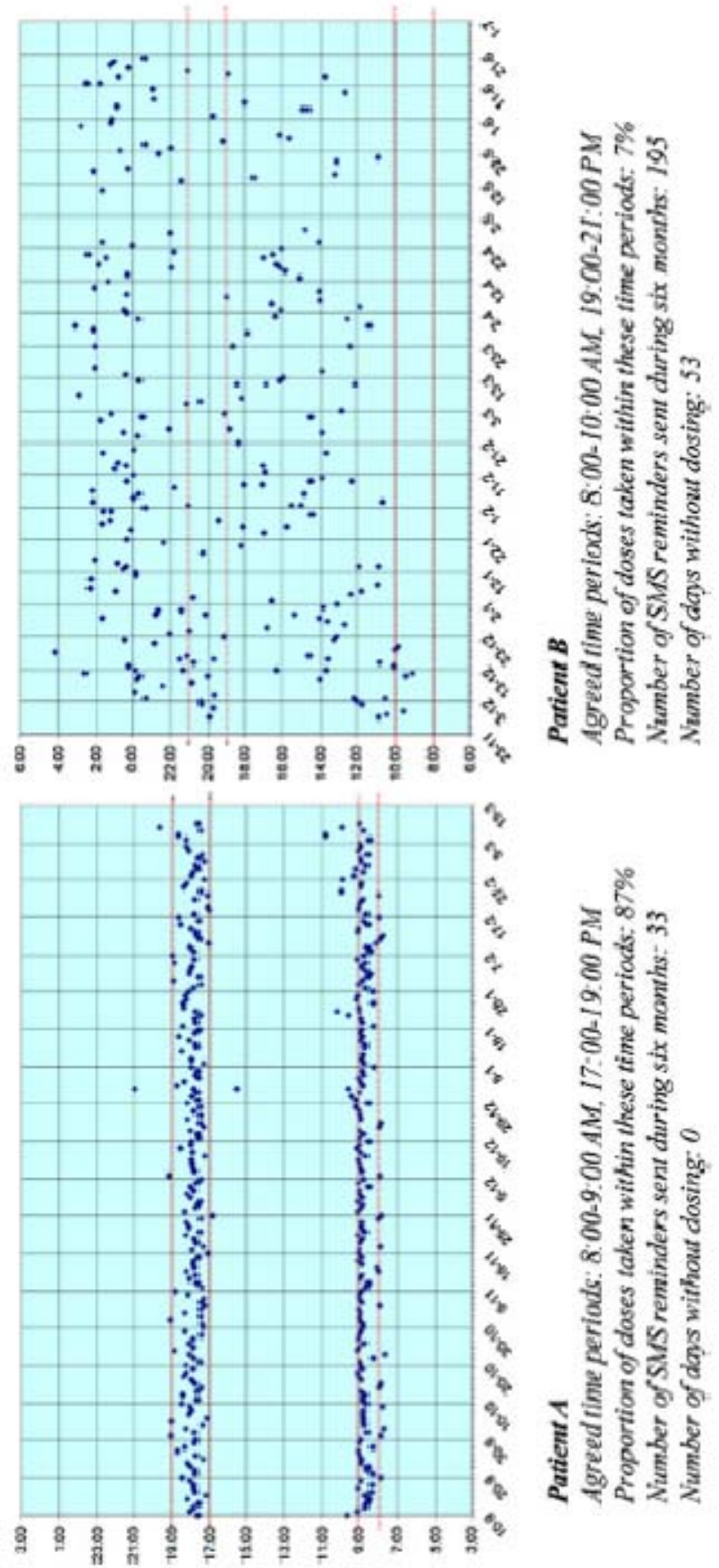
Vervloet, M., Dijk, L. van, Santen-Reestman, J., Vlijmen, B. van, Wingerden, P. van, Bouvy, M.L. Bakker, D.H. de. SMS reminders improve adherence to oral medication in type 2 diabetes patients who are real time electronically monitored. International Journal of Medical Informatics: 2012, 81(9), 594-604

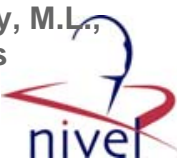

Table 1 - Characteristics of participants in the SMS group and control group at baseline."

\begin{tabular}{|c|c|c|}
\hline & SMS group $(n=56)$ & Control group $(n=48)$ \\
\hline Age (years), mean $\pm \mathrm{SD}$ & $54.9 \pm 6.6$ & $54.6 \pm 6.9$ \\
\hline Male, $n(\%)$ & $31(55.4)$ & $26(54.2)$ \\
\hline \multicolumn{3}{|l|}{ Education level, $n(\%)$} \\
\hline Low & $17(30.4)$ & $17(35.4)$ \\
\hline Medium & $18(32.1)$ & $10(20.8)$ \\
\hline High & $11(19.6)$ & $12(25.0)$ \\
\hline Missing & $10(17.9)$ & $9(18.8)$ \\
\hline \multicolumn{3}{|l|}{ Ethnicity, $n(\%)$} \\
\hline Native Dutch & $29(51.8)$ & $26(54.2)$ \\
\hline Western immigrant & $3(5.4)$ & $2(4.2)$ \\
\hline Non-Western immigrant & $15(26.8)$ & $12(25.0)$ \\
\hline Missing & $9(16.1)$ & $8(16.7)$ \\
\hline Self-reported insulin use, $n(\%)$ & $15(26.8)$ & $12(25.0)$ \\
\hline \multicolumn{3}{|l|}{ Other self-reported co-medication, $n$ (\%) } \\
\hline Lipid lowering medication & $29(51.8)$ & $16(33.3)$ \\
\hline Antihypertensive medication & $27(48.2)$ & $25(52.1)$ \\
\hline Blood thinners & $11(19.6)$ & $6(12.5)$ \\
\hline Benzodiazepines & $8(14.3)$ & $7(14.6)$ \\
\hline Asthma medication & $3(5.4)$ & $2(4.2)$ \\
\hline Rheumatoid arthritis medication & $5(8.9)$ & $2(4.2)$ \\
\hline \multicolumn{3}{|l|}{ Medication regimen, $n(\%)$} \\
\hline One daily dose & $11(19.6)$ & $12(25.0)$ \\
\hline Two daily doses & $25(44.6)$ & $27(56.3)$ \\
\hline Three daily doses & $20(35.7)$ & $9(18.8)$ \\
\hline Baseline refill adherence $(\%)$, mean \pm SD & $61.5 \pm 14.1$ & $62.2 \pm 12.8$ \\
\hline \multicolumn{3}{|l|}{ Length agreed time period (min), mean $\pm \mathrm{SD}$} \\
\hline First time period & $78 \pm 38$ & $70 \pm 42$ \\
\hline Second time period (if two daily doses) & $72 \pm 27$ & $68 \pm 30$ \\
\hline Third time period (if three daily doses) & $74 \pm 27$ & $67 \pm 20$ \\
\hline
\end{tabular}

Table 2 - Adherence to oral antidiabetics (quality of execution) of patients in the SMS group and control group calculated over the length of the intervention period.

\begin{tabular}{|c|c|c|c|}
\hline & SMS group $(n=56)$ & Control group $(n=48)$ & $p$-Value \\
\hline Days without dosing, mean $n \pm \mathrm{SD}$ & $11.9 \pm 18.8$ & $13.8 \pm 14.5$ & 0.283 \\
\hline Missed doses, mean $\% \pm \mathrm{SD}$ & $14.5 \pm 15.7$ & $19.2 \pm 16.0$ & 0.065 \\
\hline Doses taken within agreed time period ${ }^{a}$, mean $\% \pm S D$ & $56.7 \pm 23.8$ & $43.2 \pm 26.2$ & 0.003 \\
\hline \multicolumn{4}{|l|}{ Doses taken within standardized window, mean $\% \pm$ SD } \\
\hline $1 \mathrm{~h}$ & $50.3 \pm 22.8$ & $38.7 \pm 23.0$ & 0.006 \\
\hline $2 \mathrm{~h}$ & $71.4 \pm 23.8$ & $57.2 \pm 23.4$ & 0.002 \\
\hline $3 \mathrm{~h}$ & $77.3 \pm 21.6$ & $65.3 \pm 22.6$ & 0.004 \\
\hline $4 \mathrm{~h}$ & $80.5 \pm 20.5$ & $70.2 \pm 21.6$ & 0.007 \\
\hline
\end{tabular}


Vervloet, M., Dijk, L. van, Santen-Reestman, J., Vlijmen, B. van, Wingerden, P. van, Bouvy, M.L. Bakker, D.H. de. SMS reminders improve adherence to oral medication in type 2 diabetes patients who are real time electronically monitored. International Journal of Medical Informatics: 2012, 81(9), 594-604
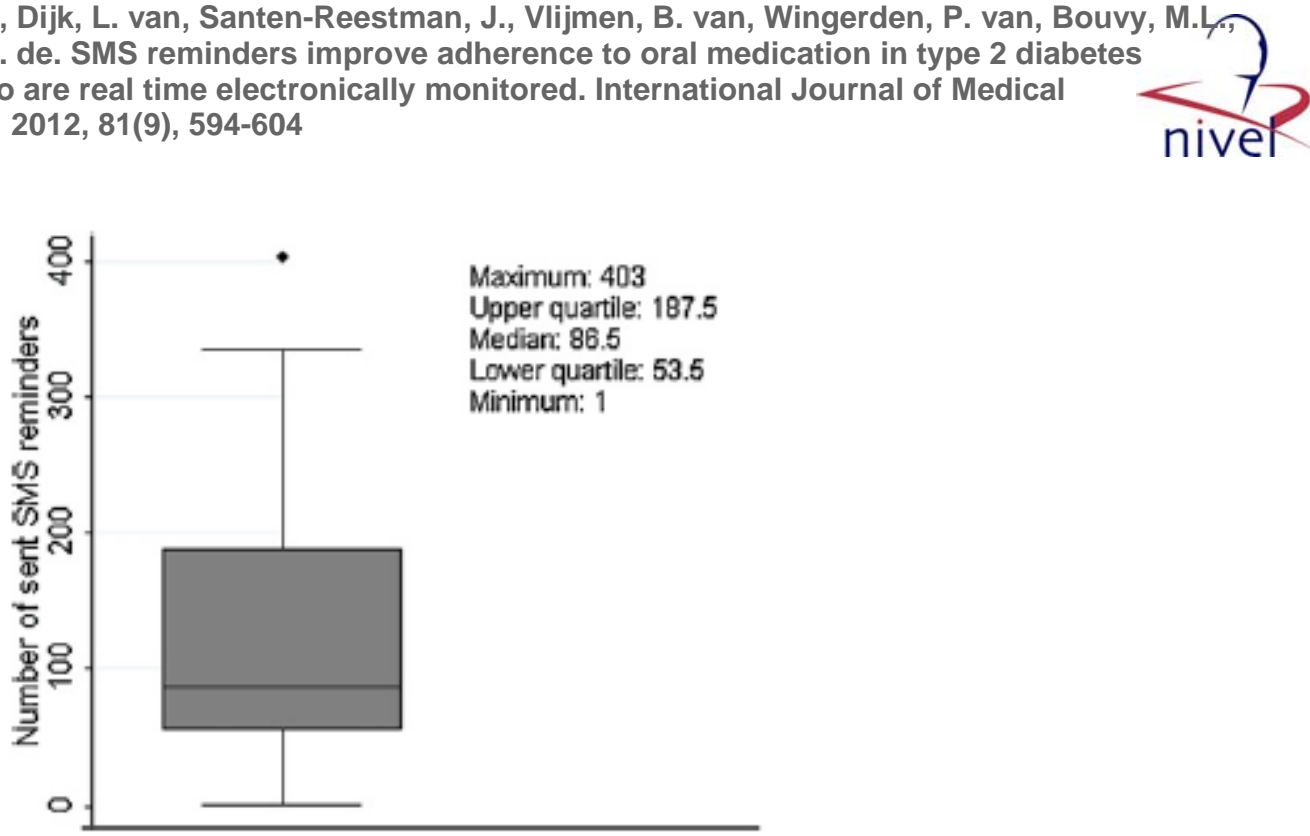

Fig. 3 - Boxplot of the number of SMS reminders sent to patients in the SMS group $(n=56)$.

Table 3 - Patients' reported awareness of their medication intake and accuracy in taking their medication since use of RTMM.

\begin{tabular}{llll} 
Patients' own judgement & SMS group & Control group & $p$-Value \\
\hline Awareness of medication use, $n(\%)$ & $(n=35)$ & $(n=33)$ \\
Higher & $15(42.9)$ & $6(18.2)$ \\
Not higher nor lower & $19(54.3)$ & $26(78.8)$ \\
Lower & $1(2.9)$ & $1(3.0)$ \\
Accuracy in taking medication, $n(\%)$ & $(n=36)$ & $(n=34)$ \\
Better & $18(50.0)$ & $9(26.5)$ \\
Not better nor worse & $15(41.7)$ & $22(64.7)$ \\
Worse & $0(-)$ & $0(-)$ \\
No idea & $3(8.3)$ & $3(8.8)$ \\
Patients' experiences with SMS reminders & & -9 \\
(Totally) agree, $n(\%)$ & & \\
It is good to know I am reminded if needed $(n=35)$ & $29(82.9)$ & \\
SMS reminders support me in medication use $(n=36)$ & $27(75.0)$ & \\
I do not react to the SMS reminders $(n=33)$ & $6(18.2)$ & \\
SMS reminders are disturbing $(n=34)$ & $7(20.6)$ & \\
SMS reminders are useful $(n=35)$ & $23(65.7)$ & \\
\hline a Patients in the control group received no SMS reminders. & & \\
\hline
\end{tabular}

